\section{Author Correction: Histidine catabolism is a major determinant of methotrexate sensitivity}

https://doi.org/10.1038/s41586-021-03487-2

Published online: 11 January 2022

Correction to: Nature https://doi.org/10.1038/s41586-018-0316-7

Published online 11 July 2018

Check for updates

Naama Kanarek, Heather R. Keys, Jason R. Cantor, Caroline A. Lewis, Sze Ham Chan, Tenzin Kunchok, Monther Abu-Remaileh,

Elizaveta Freinkman, Lawrence D. Schweitzer \& David M. Sabatini

In Fig. If of this Article, one of the three biological replicates of the control drug doxorubicin was erroneously duplicated from another experiment. The original Fig. If is shown next to the corrected panel without the erroneous replicate as Fig. 1 of this Amendment. The Source Data for the original Fig. 1, which had included erroneous data in the tab survival MTX' regarding this doxorubicin replicate, has also been corrected and is shown as Supplementary Information to this Amendment. In the Fig. 1 legend, the text “...compared to wild-type cells $(n=3$, biological replicates," should have read:'...compared to wild-type cells $(n=3$, biological replicates, except for doxorubicin in Ramos cells, where $n=\mathbf{2 ,}$,.
In Extended Data Fig. 11 of this Article, two of the histological sections in the 'methotrexate' group (top and bottom images in Extended Data Fig. 11a) and two sections in the 'vehicle' group (middle and bottom images in Extended Data Fig. 11b) were duplicated, and the sections in the 'histidine supplementation' (bottom image in Fig. 11b) and 'methotrexate' (top image in Extended Data Fig. 11b) groups were also duplicated. The original panels of Extended Data Fig. 11 are shown next to the corrected panels as Fig. 2 of this Amendment. In Supplementary Fig. 3 of this Article, two of the histological sections in the 'methotrexate' group were duplicated. The original and corrected Supplementary Fig. 3 is shown as Supplementary Fig. 1 to this Amendment, with the new panels marked by red outlines. There were errors in the Source Data of Fig. 2 that have been corrected and have been highlighted in red in Supplementary Information to this Amendment; the data in the original Fig. 2 were correct. In the Methods section, the reference for the sgRNA library that was used for the screen (Wang et al. (2014), ref. 7) was incorrect, and the updated library was instead from Wang et al. $(2015)^{1}$. The library can also be found here: http://www.addgene.org/ pooled-library/sabatini-crispr-human-high-activity-3-sublibraries/. These errors do not affect the conclusions of the paper, and the original Article has not been corrected online.

David Sabatini is no longer affiliated with the Whitehead Institute or the Howard Hughes Medical Institute. At the request of the Whitehead Institute and to ensure execution of the duties of corresponding author, the corresponding author on this Author Correction is now Naama Kanarek (naama.kanarek@childrens.harvard.edu),replacing David Sabatini. All authors agree with the Author Correction and the change of the corresponding author.

Supplementary information is available in the online version of this Amendment.

1. Wang, T. et al. Identification and characterization of essential genes in the human genome. Science 350, 1096-1101 (2015).

(c) The Author(s), under exclusive licence to Springer Nature Limited 2022
Original published Fig. $1 f$

$\mathbf{f}$

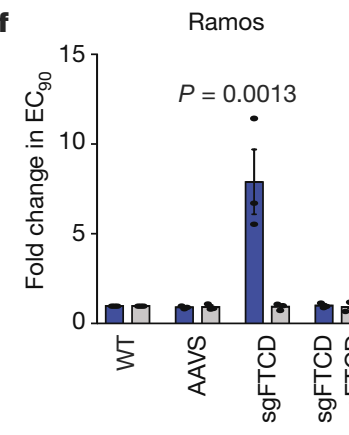

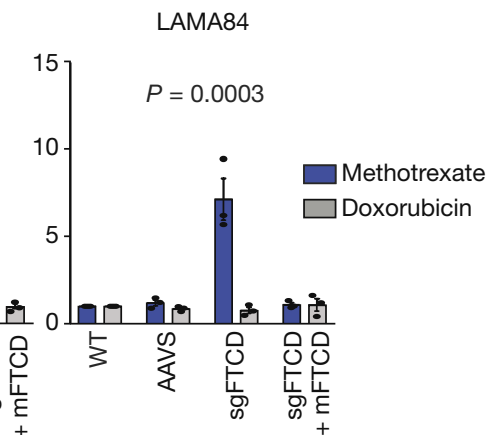

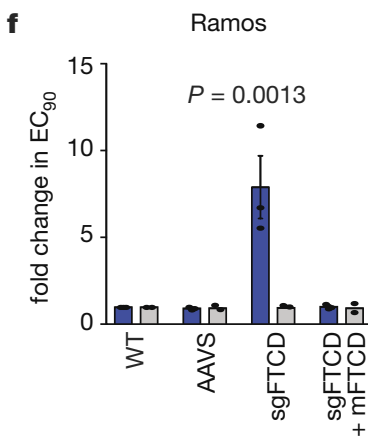

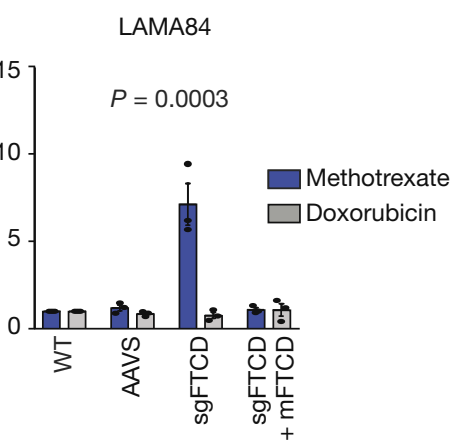

Fig. 1 | This is the original published Fig. If (left) and the corrected Fig. If (right) of the original Article. 


\section{Corrections \& amendments}

\section{Original published Extended Data Fig. 11}

a

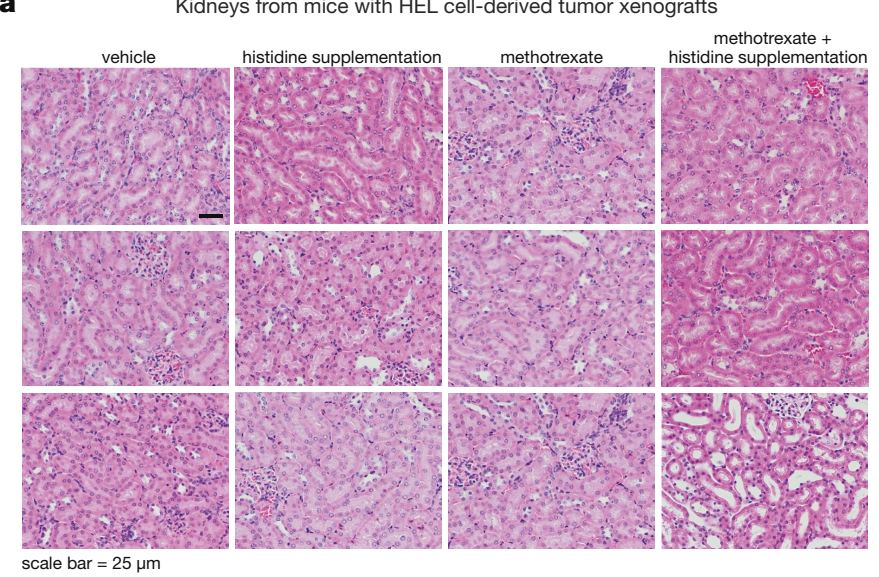

b
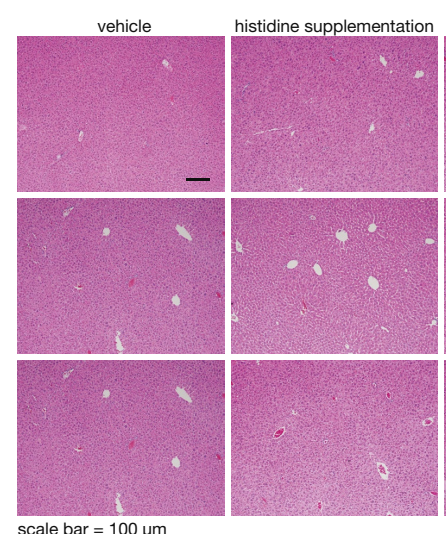

scale $\mathrm{bar}=100 \mu \mathrm{m}$

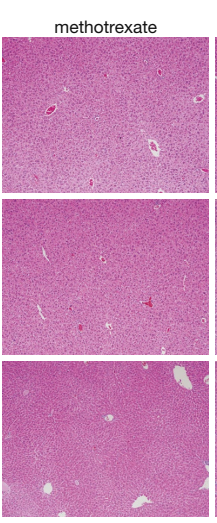

methotrexate +
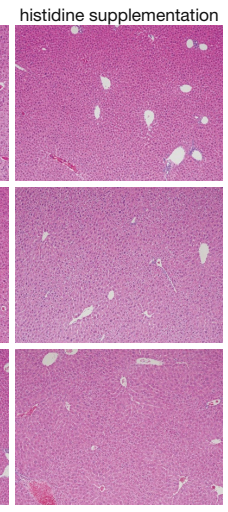

Fig. 2 This is the original published Extended Data Fig. 11 and the corrected Extended Data Fig. 11 of the original Article. The new panels are marked by red outlines.

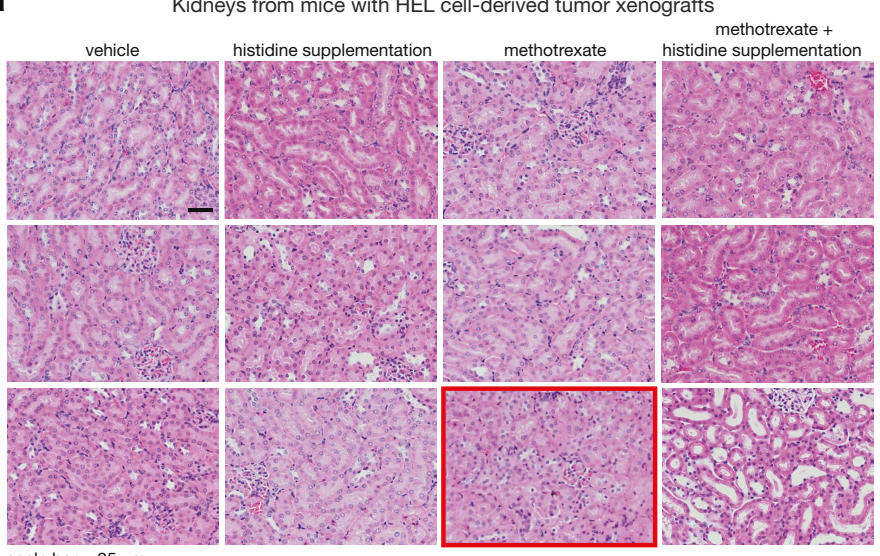

scale bar $=25 \mu \mathrm{m}$

b

Livers from mice with HEL cell-derived tumor xenografts

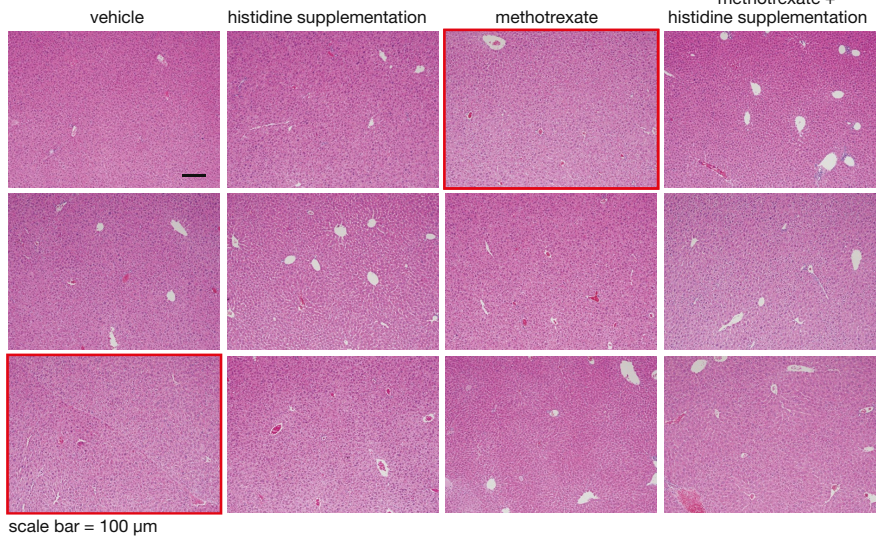

